\title{
Extreme-ultraviolet coherent pulse amplification in argon
}

\author{
C. Serrat ${ }^{*}$ \\ Department of Physics, Polytechnic University of Catalonia, Colom 11, E-08222 Terrassa (Barcelona), Spain \\ J. Seres and E. Seres \\ Institute of Atomic and Subatomic Physics, Vienna University of Technology, Stadionalle 2, A-1020 Vienna, Austria \\ S. Namba \\ Graduate School of Engineering, Hiroshima University, 1-4-1 Kagamiyama, Higashi-Hiroshima, Hiroshima 739-8527, Japan
}

(Received 21 March 2019; published 25 June 2019)

\begin{abstract}
The amplification of ultrashort extreme-ultraviolet (XUV) pulses in argon in high-order harmonic generation processes is studied by using the time-dependent Schrödinger equation in the spin-free one-active-electron and single-atom approximation. We consider a neutral argon atom initially in the valence $3 p$ state and a sufficiently intense two-cycle driving infrared (IR) pulse for the atom to be mainly ionized after the first laser cycle. The correlated dynamics and transitions from the valence $3 p$ to a virtual subvalence $3 \mathrm{~s}$ state and in the ionized regions are examined by synchronizing a 1.5 -fs XUV pulse to the IR pulse. The calculated single-atom gain spectrum (26-45 eV) agrees with recent experimental measurements. We discuss different channels that can be present in the gain process as a function of pulse parameters and through an analysis of the dynamics of the populations in terms of field-free eigenstates. When the XUV pulse is considered at the end of the driving IR field the amplification is due to contributions of stimulated recombination from excited Rydberg and low energy continuum states to the $3 \mathrm{~s}$ and $3 \mathrm{p}$ states of argon. In regions where the IR field is intense, high energy and angular momentum states are populated and the medium can interact with the pulses through bound and continuum states involving parametric transitions, which is further confirmed by studying classical electron trajectories. We discuss how these parametric interactions might be suitable for amplification of photon energies far from the ionization threshold as observed in the experiments.
\end{abstract}

DOI: 10.1103/PhysRevA.99.063425

\section{INTRODUCTION}

The amplification of extreme-ultraviolet (XUV) and x-ray radiation in high-order harmonic generation (HHG) processes has been largely studied in previous years because of the importance of enhancing the associated HHG low conversion efficiency and for understanding the generation process more accurately. This improvement is key for the use of table-top coherent XUV and $\mathrm{x}$-ray sources in many applications $[1,2]$. In particular, simultaneous excitation of atoms with an intense infrared (IR) driving pulse and a weak XUV pulse or train of pulses conveniently synchronized has been extensively investigated both theoretically and experimentally as a method to amplify the signal from the single-atom level interaction and also considering the effects of propagation [3-21].

Ultrashort XUV pulses of central frequencies far from the ionization threshold were successfully amplified in experiments using helium gas $[8,9,12,13,15]$. The fundamental physics behind the involved scattering mechanisms, however, lack a detailed explanation which is certainly very necessary for the possibility of amplifying XUV and $\mathrm{x}$-ray coherent pulses at any desired photon energies. Different theoretical

\footnotetext{
*carles.serrat-jurado@upc.edu
}

approaches $[9,10,12,14,15,17]$ have described the amplification in terms of extended electron trajectories in the HHG process together with parametric interactions, such as X-ray parametric amplification (XPA) and intra-XUV-pulse parametric amplification (IXPA), although only qualitative agreement with the measurements has been reported so far $[8,9,12,13]$. Some of these studies have been performed in the frame of the solution of the time-dependent Schrödinger equation (TDSE) both in the strong-field (SFA) and in the single-activeelectron (SAE) approximations. Other faithful theories also based on $a b$ initio simulations have considered the need of population inversion to overcome the possible loss channels in the medium [19], leading to some weak amplification at the lower photon energies only close to the ionization threshold. Previously in Refs. [15,17] enhancement of high photon energies far from the ionization threshold in accordance with the measurements was achieved on the basis of parametric and intra-XUV-pulse parametric processes, but this was not accounted for at all in Ref. [19] and amplification any distant from the ionization threshold as observed in the experiments is there missing. A single-electron theoretical study has also been reported in the basis of angular momentum dependent potentials for the $\mathrm{Li}^{+}$ion and a comparison between onedimensional (1D) and three-dimensional (3D) simulations has been performed [20], although no intent to compare to the experimental results is realized. 

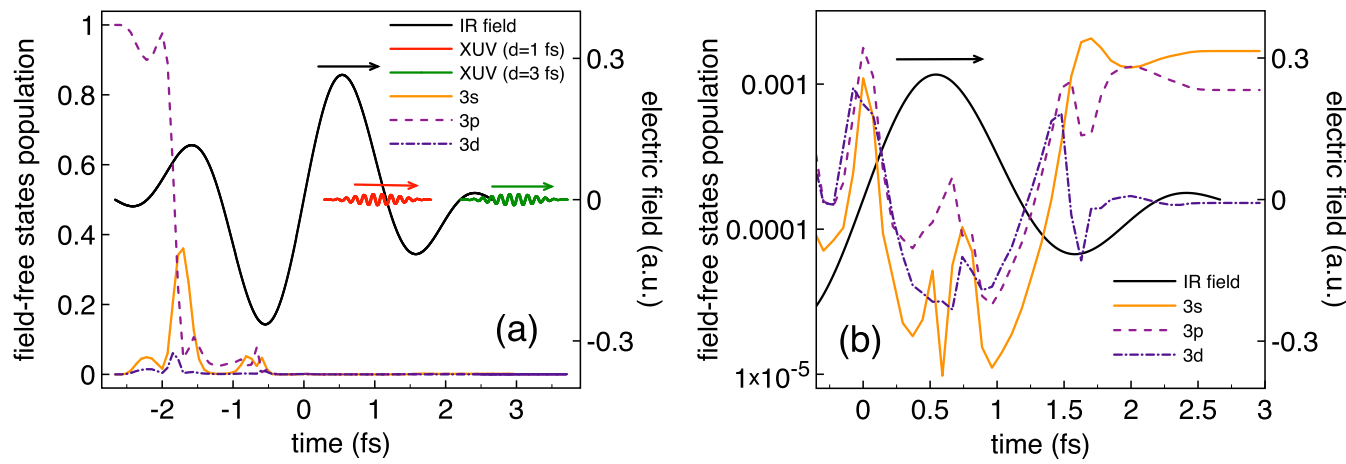

FIG. 1. We consider the IR field resulting from a 800 -nm two-cycle $\cos ^{2}$ vector potential interacting with the argon atom together with a XUV weak pulse synchronized with the IR field at different delays. (a) The IR field with peak intensity of $5 \times 10^{15} \mathrm{~W} / \mathrm{cm}^{2}$ is shown to produce a large ionization of the model atom, which is considered initially in the ground $3 p$ state. Examples of two weak XUV pulses at two different delays are also shown. (b) The population in the $3 \mathrm{~s}, 3 \mathrm{p}$, and $3 \mathrm{~d}$ field-free states is plotted in the time interval the XUV pulse interacts.

In the present study we examine the simultaneous excitation of argon atoms with an intense IR pulse and a weak XUV pulse in the SAE approximation. We numerically solve the spin-free SAE [22,23] 3D-TDSE using the implementation described in Ref. [24], with an effective potential for the argon atom as given in Ref. [22]. The model that we consider can only accommodate one electron which in our simulations is initially at the argon ground state $3 \mathrm{p}$. All the inner states (1s, $2 \mathrm{~s}, 2 \mathrm{p}, 3 \mathrm{~s}$ ) are therefore initially empty in the model and can be filled. We have checked that no population is transferred to the more inner shells $(1 \mathrm{~s}, 2 \mathrm{~s}, 2 \mathrm{p})$ of the neutral atom during the interaction with the IR pulse, but some population transfer to the $3 \mathrm{~s}$ state is observed in the calculations. We hence analyze the regions where the $3 p$ state of argon has been mainly ionized and the $3 \mathrm{~s}$ state is also almost empty, which is a feasible configuration as it will be further detailed below. Certainly, while tunnel ionization [25] of argon predicts one order of magnitude lower ionization rate for $3 \mathrm{~s}$ states than for the $3 p$ states due to the higher ionization energy of $3 \mathrm{~s}$ $(28.3 \mathrm{eV})$ compared to $3 \mathrm{p}(15.8 \mathrm{eV})$, the inner shell $3 \mathrm{~s}$ state can still give an essential contribution to the different amplifying processes that we are describing. The $3 \mathrm{~s}$ state can be depleted by several ionization and excitation channels such as multiphoton processes and excitation beyond tunnel ionization. Those additional mechanisms can be expected from experiments [26] measuring a high rate of multiple ionized argon at intensities $\sim 10^{15} \mathrm{~W} / \mathrm{cm}^{2}$, with the second ionization energy $(27.6 \mathrm{eV})$ being comparable to the ionization energy of the $3 \mathrm{~s}$ state. The interaction geometry of our analysis is shown in Fig. 1(a), where we plot the IR field together with two examples of XUV pulses synchronized with the IR field at two different delays. As shown in Fig. 1(a), $t=0$ is considered at the center of the sin-IR pulse so that the delay between the $\mathrm{XUV}$ and the IR pulse is given by the central time position of the XUV pulse. As commented above, the atom is initially in the state 3p. The population of this state is plotted as a function of time in Fig. 1(a) for a driving IR pulse with a peak intensity of $5 \times 10^{15} \mathrm{~W} / \mathrm{cm}^{2}$. The state populations are computed by projecting the electron wave function onto the field-free eigenstates of the Hamiltonian model, as it is further detailed below. As can be seen in Fig. 1(a) the population decreases to very small values after the first IR cycle. The population in $3 \mathrm{~s}$, initially empty in the atom model, becomes virtually filled and finally also depleted at $t=0$, and this is also the case for the excited state $3 \mathrm{~d}$. The ionization of the atom at $t=0$ is therefore considered as the optimal condition for the goal of our study. Figure 1(b) shows the small populations of the three bounded states $3 \mathrm{~s}, 3 \mathrm{p}$, and $3 \mathrm{~d}$ that result as a consequence of the interaction or the intense two-cycle IR pulse with the atom model in the delay region of our interest $t>0$. The study that we perform is therefore based on the interaction of the IR and XUV pulses in regions where the bound populations in the spin-free states $3 \mathrm{~s}, 3 \mathrm{p}$, and $3 \mathrm{~d}$ is $\sim 0.001 \%-0.2 \%$, depending on the case. We study different central frequencies of the XUV pulse and consider several values of the IR peak intensity. In particular, we study the 26-45 eV XUV-gain region observed in recent HHG measurements in argon, which is distant from the argon first ionization threshold $(15.75 \mathrm{eV})$, and we investigate single-atom scattering mechanisms that are involved in the XUV pulse amplification.

The paper is organized as follows: In Sec. II we examine single-atom XUV transient absorption signals [27] by varying the delay between a weak XUV pulse and a strong IR pulse in the delay region that includes the second cycle of the IR pulse $t>0$; these are the main results of our simulations. We then compare our results with recent experiments performed in argon. We show that the SAE spin-free model correctly describes the gain spectral region observed in the experimental measurements $(26-45 \mathrm{eV})$. We later analyze in detail some more specific regions to illustrate the physical mechanisms involved in the amplification processes. We do this by performing a study both in the frequency and in the time domains: the frequency-resolved probe absorption signal of the XUV pulses is investigated in Sec. III. In Sec. IV we present a study of the evolution in time of the electron wave function in terms of field-free eigenstates, which gives essential information about the instantaneous population distribution and dynamics resolved by the $n$ quantum number, the $L$ angular momentum channel, and the eigenenergy $E$ of the states. Definitely, the combined study in the frequency and time domains allows us to identify how amplification processes due to correlated transitions from both bound and in the continuum excited states to the $3 \mathrm{~s}$ and $3 \mathrm{p}$ eigenstates of argon are produced in regions where the intensity of the IR pulse is sufficiently low. In the regions where the IR field is moderate and strong high energy level and angular 

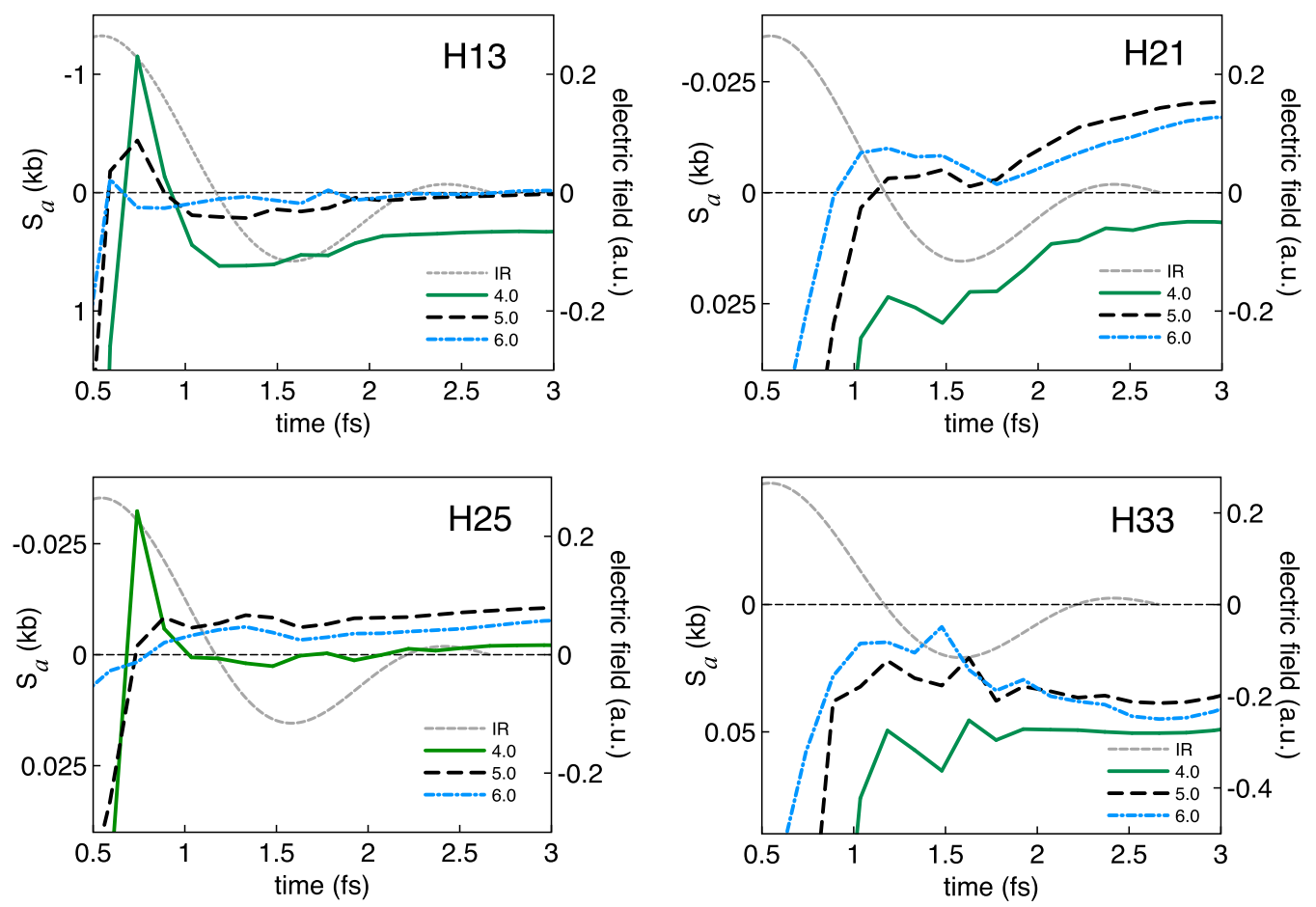

FIG. 2. Spectrally integrated XUV probe absorption signal $S_{a}$ [see Eq. (2)] as a function of the delay of the XUV pulse with respect to the IR pulse. Negative values show the total XUV gain regions while positive values show absorption regions. The peak IR field intensities in terms of $10^{15} \mathrm{~W} / \mathrm{cm}^{2}(4.0,5.0$, and 6.0) are indicated in the subplots and by the different styles and colors of the lines. The central frequencies of the XUV pulse considered are shown as H13-H33 in each subplot, Hn meaning the corresponding n harmonic of an 800-nm field. The light gray-dashed line is shown for clarity and corresponds to an example of the IR field, with $3 \times 10^{15} \mathrm{~W} / \mathrm{cm}^{2}$ (see values in the right axis in a.u.).

momentum states in the continuum are populated and both bound-continuum and continuum-continuum [28-30] intraXUV-pulse transitions can be significant. We further confirm that IXPA and XPA parametric channels are open in this region by studying electron trajectories in the frame of the semiclassical three-step theory [31]. In Sec. V we discuss the results in our work and conclude that parametric IXPA processes involving bound and continuum states together with the more regular XPA processes are plausible candidates for amplification of XUV pulses with photon energies far distant from the ionization threshold, as it was reported for the experiments in helium $[8,9,12,13,15]$.

\section{MAIN NUMERICAL RESULTS AND COMPARISON WITH THE EXPERIMENTS}

Figure 2 shows the total probe absorption signal obtained by varying the delay between the XUV and the IR pulses. We have considered a linearly polarized IR field resulting from a two-cycle $\cos ^{2}$ envelope vector potential of $800 \mathrm{~nm}$, with IR field peak intensities of $4.0-6.0 \times 10^{15} \mathrm{~W} / \mathrm{cm}^{2}$, as indicated in the subplots in Fig. 2. The IR field interacts with an argon atom together with a weak $\left(\sim 10^{12} \mathrm{~W} / \mathrm{cm}^{2}\right)$ XUV field of 1.5 -fs total $\cos ^{2}$ envelope duration, considering the harmonic frequencies H13-H33, as shown in the figure (see also Fig. 1).

To study the absorption of the XUV pulse in the medium, we compute the absorption spectrum $S(\omega)$ in the frame of transient absorption signals as described in Ref. [27]

$$
S(\omega)=\frac{\omega_{X} \operatorname{Im}\left[E_{X}^{*}(\omega) D_{X}(\omega)\right]}{\epsilon_{0} c \int d \omega\left|E_{X}(\omega)\right|^{2}},
$$

where $\omega_{X}$ is the central frequency of the XUV pulse, $E_{X}(\omega)$ represents its complex spectrum, $D_{X}(\omega)=D(\omega)-D_{\mathrm{IR}}(\omega)$, where $D(\omega)$ is the spectrum obtained from the acceleration of the dipole when the system is excited with the combined IR+XUV pulses, and $D_{\mathrm{IR}}(\omega)$ is the one calculated with the IR field alone. In this way, $S(\omega)$ provides the absorption that a weak probe pulse $E_{X}(\omega)$ would experience interacting with a system that has been pumped and presents a dipole acceleration given by $D_{X}(\omega)$. The total probe absorption signal is then obtained by integration of $S(\omega)$,

$$
S_{a}=\int_{0}^{\infty} d \omega S(\omega) .
$$

Positive values of $S(\omega)$ or $S_{a}$ mean absorption of the XUV pulse in the single-atom medium, while negative values mean amplification (gain). The absorption cross section $S_{a}$ multiplied by the number density of atoms results in the absorption or gain coefficient [27]. $S(\omega)$ in Eq. (1) is written in SI units $\left(\mathrm{m}^{2} \mathrm{~s}\right)$, with $\epsilon_{0}$ and $c$ being the vacuum permittivity and the speed of light, respectively. In all simulations atomic units (a.u.) are considered, however, and in some cases we also use $S(\omega)$ in b (barn)/eV and $S_{a}$ in units of area b. Importantly in our study, we describe relevant cases where $S(\omega)$ is positive in a part of the XUV spectral region while it is negative in other parts, which, as we will further discuss below, will lead us to examine how transparency can be considered in terms of the single-atom total absorption $S_{a}$.

Different regions with $S_{a}<0$ can be seen in Fig. 2. These regions hence correspond to the different delays between the XUV and the IR pulses at which amplification of the XUV 

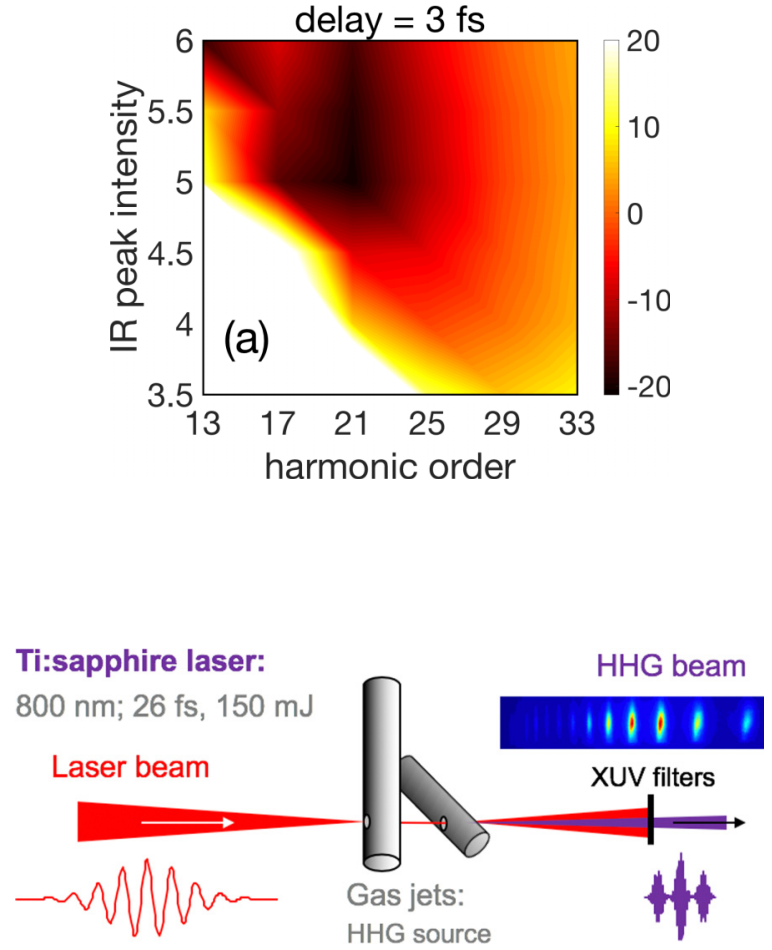
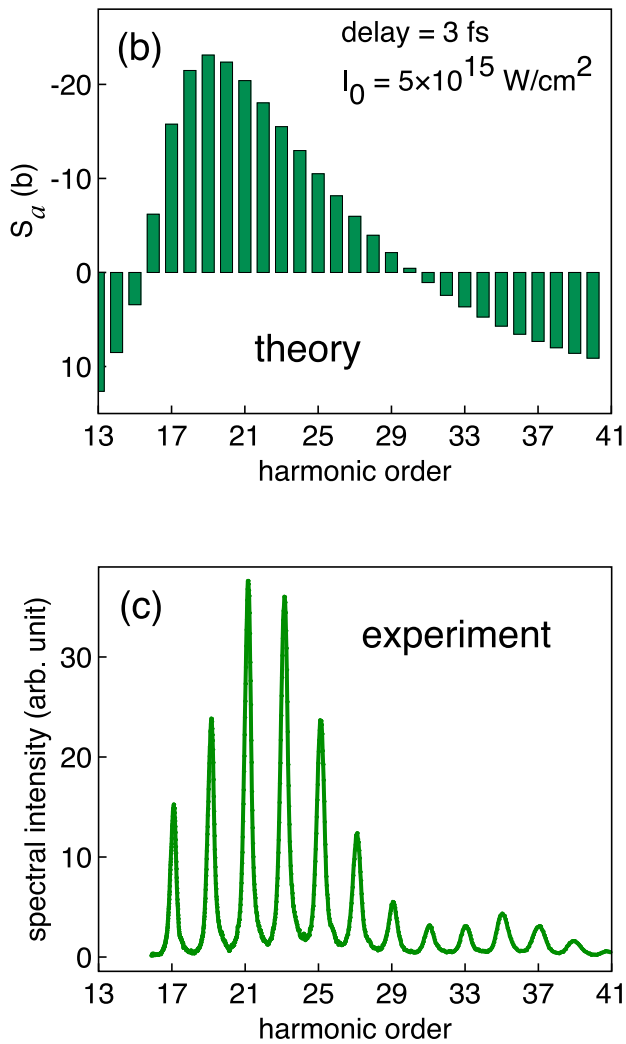

FIG. 3. (a) Spectrally integrated single-atom XUV absorption signal $S_{a}$ (in barns) as a function of harmonic order and the IR peak intensity (in terms of $10^{15} \mathrm{~W} / \mathrm{cm}^{2}$ ). The delay of the XUV pulse is in this case $t=3 \mathrm{fs}$. (b) $S_{a}$ for $5 \times 10^{15} \mathrm{~W} / \mathrm{cm}^{2}$, as obtained from a cut of (a). Note that the vertical axis is reversed for a clearer comparison with the measurements. (c) Experimental results obtained with an IR peak intensity of $\sim 5 \times 10^{14} \mathrm{~W} / \mathrm{cm}^{2}$ and of 26-fs duration (FWHM).

pulse is obtained in the single-atom case. In the case of H13 $(20.15 \mathrm{eV})$, the peak of negative absorption (gain) obtained at the high IR field region ( $t \approx 0.75 \mathrm{fs}$ ) is due to stimulated transitions from IR-excited states to the valence $3 p$ state [32], and we have checked that this effect increases as the XUV harmonic is considered closer to the argon first ionization threshold $(15.75 \mathrm{eV})$. Amplification of harmonics close to the first ionization threshold however is not the intention of the present work and will be analyzed elsewhere. For the larger harmonics $(\mathrm{H} 21-\mathrm{H} 25)$, other relative maxima in the gain curves and absorption areas are obtained and some will be examined in detail here in this study. In that respect, the strength of the IR electric field is decisive for the processes involved in the different delays since it sets the shift of the excited states and therefore changes the available resonances for stimulated emission.

In Figs. 3(a) and 3(b) the total absorption $S_{a}$ at the XUV delay of $3 \mathrm{fs}$ is shown. In this case, the XUV field interacts only partially with the atom in a region where the IR field is already weak (see also Fig. 1). Figure 3(a) shows the spectrally integrated XUV absorption signal $S_{a}$ (in barns) as a function of the harmonic order and the IR peak intensity (in terms of $10^{15} \mathrm{~W} / \mathrm{cm}^{2}$ ). For clarity, the colors in the figure have been saturated at $S_{a}=20$ b. In Fig. 3(b) a horizontal cut of Fig. 3(a) at $5 \times 10^{15} \mathrm{~W} / \mathrm{cm}^{2}$ is shown. In the experimental conditions, the sign of the total absorption $S_{a}$ strongly affects the shape of the generated harmonic spectrum due to propagation effects. A generated spectrum can be estimated by $I(\omega) \sim\left(e^{-S_{a} \rho Z}-\right.$ $\left.2 e^{-S_{a} \rho Z / 2} \cos (\Delta k Z)+1\right) /\left((\Delta k Z)^{2}+\left(S_{a} \rho Z / 2\right)^{2}\right)$, where $\rho$ is the number density of atoms, $Z$ is the length of the medium, and $\Delta k$ is the phase mismatch [8,9]. This expression in accordance with our simulations [Fig. 3(b)] predicts absorptionlimited HHG for $S_{a}>0$, in the ranges before H15 and beyond H31, and an enhanced signal if $S_{a}<0$ (gain) between $\mathrm{H} 17$ and H29, which is in a very good agreement with the experimental spectrum shown in Fig. 3(c). Other results of the measurement series will be published elsewhere. In the mentioned experiment, shown in Fig. 3, 150-mJ, 26-fs pulses of a Ti:sapphire laser system were loosely focused with a mirror having focal length of $6 \mathrm{~m}$ supporting a peak intensity up to $10^{15} \mathrm{~W} / \mathrm{cm}^{2}$ in the focus. The high-order harmonic source was constructed as a sequence of two jets with adjustable distance and position similarly to $[13,15]$. The interaction lengths of the jets were $1 \mathrm{~mm}$, their distance was $20 \mathrm{~mm}$, and argon gases were used as the active medium. The generated HHG signal was measured after a 300-nm-thick Al foil used to block the laser beam. It is worth noting that the level of atom ionization and XUV amplification that we describe has also been numerically obtained with longer and weaker IR pulses in some tests. In particular, in Fig. 4 we show how a 10-cycle 800-nm flat-top IR pulse with intensity of $10^{15} \mathrm{~W} / \mathrm{cm}^{2}$ produces amplification of a H21 XUV pulse synchronized at $t=8 \mathrm{fs}$, with an integrated probe absorption of $-9.5 \mathrm{~b}$, which is comparable to the gain values obtained with the two-cycle IR pulse of peak intensity as 5-6x $10^{15} \mathrm{~W} / \mathrm{cm}^{2}$ described in Figs. 2, 3, and 5. Therefore, even 


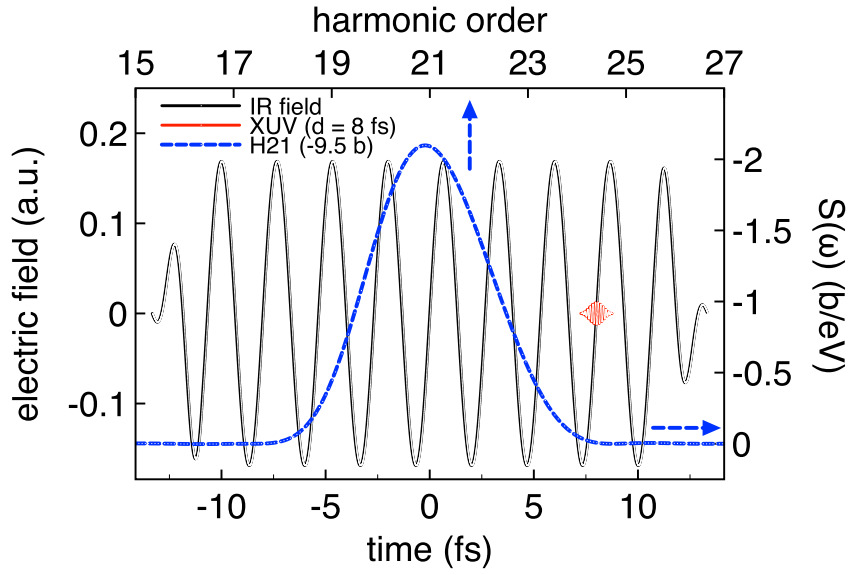

FIG. 4. Ten-cycle 800-nm flat-top IR pulse with intensity of $10^{15} \mathrm{~W} / \mathrm{cm}^{2}$ which produces amplification of an $\mathrm{H} 21 \mathrm{XUV}$ pulse synchronized at $t=8 \mathrm{fs}$ with integrated probe absorption of $-9.5 \mathrm{~b}$, as indicated. The population in the bound states after the IR pulse is $\sim 0.006 \%$.

longer pulses with intensities below $10^{15} \mathrm{~W} / \mathrm{cm}^{2}$ as used in the experiments are expected to produce similar amplification values. An extensive numerical analysis with such long pulses requires a very large amount of computer resources, however, without granting us with more information, and hence calculations with a short two-cycle pulse were performed.

\section{FREQUENCY RESOLVED ABSORPTION: UNIFORM AND MIXED INTRA-XUV-PULSE AMPLIFICATION}

In order to discern the scattering processes that might be involved in the calculated single-atom XUV amplification, we specifically study the amplification of the harmonic 21 (H21). First, we evaluate its frequency resolved absorption signal $S(\omega)$ as a function of delay for the particular value of the IR peak intensity of $6.0 \times 10^{15} \mathrm{~W} / \mathrm{cm}^{2}$. This is shown in Fig. 5. As the delay of the H21 XUV pulse is increased from $t \approx 0.8$ fs to $t \approx 3.8$ fs [Fig. 5(a)], we observe different regions of amplification which look qualitatively different. These regions are dependent on the strength of the IR field and the degree of ionization together with the characteristics of the XUV field. In Fig. 5(b) some spectra are shown at different delays, as indicated. For instance, the dotted light gray line shows the broad $\mathrm{H} 21 S(\omega)$ spectrum at a delay of $t=0.9 \mathrm{fs}$. Clearly, there is absorption about the photon energy region of $\approx 1.08-1.20$ a.u. $(\approx \mathrm{H} 19-\mathrm{H} 21$, in terms of harmonics), while the higher part of the H21 spectrum is amplified $S[(\omega)<0]$ about $\approx 1.20-1.37$ a.u. $(\approx \mathrm{H} 21-\mathrm{H} 24)$. In this case the integrated absorption gives a small net absorption of $S_{a}=0.8 \mathrm{~b}$, as it is indicated in Fig. 5(b). In other cases, e.g., the full red line at $t=1.0 \mathrm{fs}$, we observe the same feature, but now the value of $S_{a}$ is negative, so that it gives a net amplification of $9.0 \mathrm{~b}$. A similar behavior can be seen by increasing the delay ( $t=1.2,1.5 \mathrm{fs}$, different dashing orange and blue lines, with $S_{a}=-6.5,-8.3 \mathrm{~b}$, respectively). The dash-dotted black line in Fig. 5(b) shows the case at $t=1.8 \mathrm{fs}$, where the net gain is again small $\left(S_{a}=-1.9 \mathrm{~b}\right)$, which is comparable in value to the one obtained for the dotted light
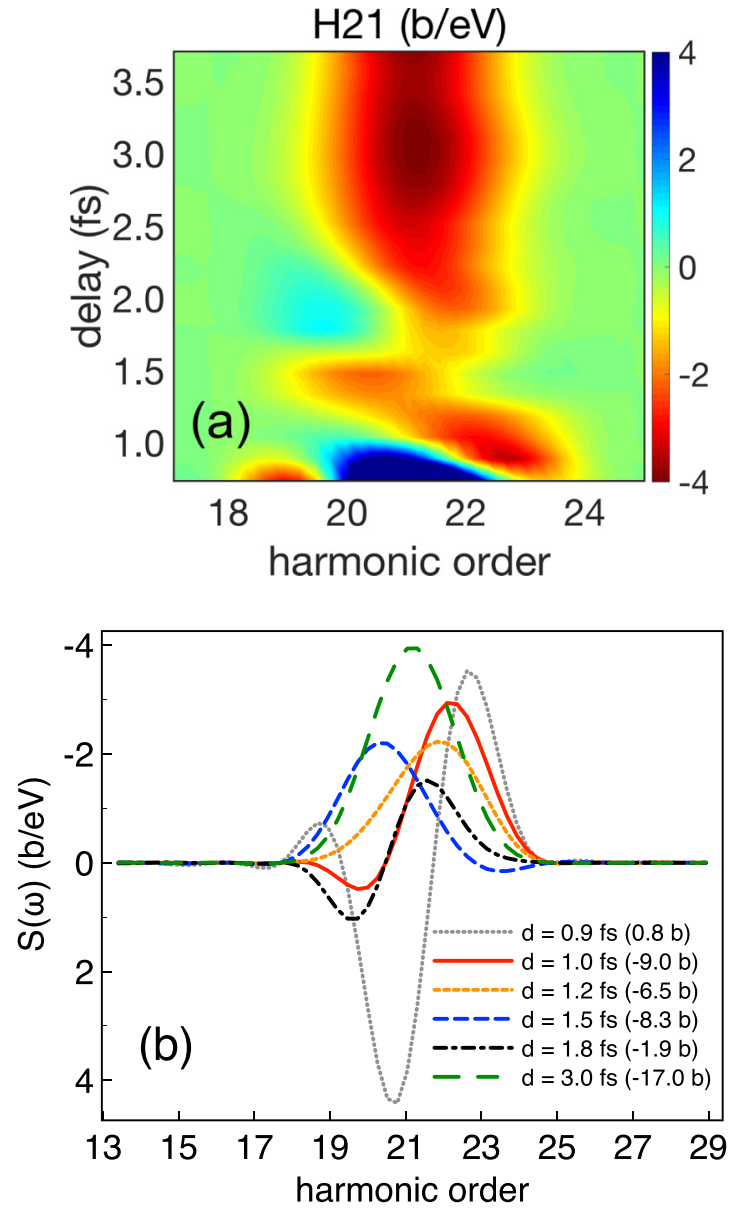

FIG. 5. (a) Frequency resolved absorption signal $S(\omega)$ of $\mathrm{H} 21$ as a function of delay for an IR peak intensity of $6.0 \times 10^{15} \mathrm{~W} / \mathrm{cm}^{2}$. (b) Some absorption spectra $S(\omega)$ of $\mathrm{H} 21$ at different delays from (a), as indicated, are shown. The integrated value $S_{a}$ is also given.

gray line $\left(S_{a}=0.8\right)$. For higher delays, the spectrum tends to a uniform amplification region, as shown by the long-dashed green line taken at $t=3.0 \mathrm{fs}\left(S_{a}=-17.0 \mathrm{~b}\right)$. At this point, we hence find it important to address the significance of the gain values $S_{a}$ that we obtain and also the meaning of XUV transparency in terms of the single-atom total absorption $S_{a}$. On the one hand, the relatively weak amplification signals that we compute are a result of single-atom calculations, and as it was clearly shown in Ref. [15], this single-atom signal when propagated in an amplifier medium results in exponential growth. Moreover, it was found in Ref. [15] that the final amplified spectral region can be shifted with respect to the single-atom central frequency gain region due to the effects of propagation. On the other hand, the particular kind of mixed-like spectral absorption and amplification signals, as the cases shown in Fig. 5(b), were reported to produce exponential growth using accurate propagated SFA computations in Ref. [15], and they were further reported and investigated in Ref. [17] as IXPA processes. It seems obvious that the value of the single-atom total probe absorption $S_{a}$ as obtained by integration of $S(\omega)$ for, e.g., the dotted light gray line and the dash-dotted black line in Fig. 5(b), although having similar total absorption $S_{a}$, might produce different effects 
once propagated in the medium, and also compared with an all spectral flat transparent signal. Therefore, we conclude that the integrated total absorption value $S_{a}$ is not sufficient to determine which geometry is going to produce amplification in a propagated scenario, where the XUV pulse reshaping in time as well as in the frequency domain is expected.

In the next section we perform a study based on the evolution in time of the electron populations in terms of fieldfree eigenstates. This investigation complements the study performed in the frequency domain and is decisive for the understanding of parametric amplification processes that are produced during the time that the XUV pulse interacts with the atom.

\section{TIME-DEPENDENT STUDY: DYNAMICS OF THE STATE POPULATIONS}

We examine the populations in the field-free eigenstates of the system during the interaction of the IR+XUV pulses for the two qualitatively different cases at $t=3.0 \mathrm{fs}$ and $t=1.0$ fs shown in Fig. 5(b) (long-dashed green and full red lines, respectively). The long-dashed green line corresponds to a case where the IR field is already weak, so that the IR-induced energy shift on the excited states is small and the absorption spectrum shows a uniform gain, while the full red line corresponds to a delay region where the IR is moderate or high during the interaction of the XUV pulse and the energy shift is correspondingly high. These two XUV delay cases are also drawn as the green and red XUV pulses at $t=3.0 \mathrm{fs}$ and $t=1.0$ fs, respectively, in Fig. 1 .

According to the absorbing-boundary TDSE simulations in the grid described in Ref. [24], and considering that the magnetic quantum number $M$ is conserved for linearly polarized light, the total time-evolving electron population in a given angular momentum channel $L$ is given by

$$
P_{L}(t)=\mathbf{L}_{L}^{T}(t) \mathbf{R}_{L}(t),
$$

where $\mathbf{L}_{L}(t)$ and $\mathbf{R}_{L}(t)$ are the left and right discretized wave functions within the angular channel $L$ at a given time $t$, respectively. The left and right eigenvectors of the fieldfree Hamiltonian are $\mathbf{U}_{L i}$ and $\mathbf{V}_{L i}$, which form a complete biorthogonal set,

$$
\sum_{i}^{N_{R}} \mathbf{V}_{L i} \mathbf{U}_{L i}^{T}=\mathbf{1},
$$

where $N_{R}$ is the number of radial grid points. Inserting Eq. (4) into the right-hand side of Eq. (3) the total population can be written as

$$
P_{L}(t)=\sum_{i}^{N_{R}} a_{L i}(t),
$$

with

$$
a_{L i}(t)=\left[\mathbf{L}_{L}^{T}(t) \mathbf{V}_{L i}\right]\left[\mathbf{U}_{L i}^{T} \mathbf{R}_{L}(t)\right] .
$$

The value $a_{L i}(t)$ is treated as the population of the field-free eigenstate $L, i$, so that the total populations in the integration grid is resolved for the quantum number $n$ and the angular momentum $L$. Indeed, the discretization used in the theory allows one to denote the bound and continuum eigenstates by a single discrete index $n$, so that regular bound states are those with negative energy eigenvalues $(E<0)$ while positive energies $(E>0)$ indicate states in the discretized continuum. Further details on the generalized eigenvalue problem considered in the simulations can be found in Ref. [24]. We analyze the time-evolving states by examining the distribution of the populations in the case that only the IR pulse interacts with the atom $\rho_{\mathrm{IR}}$ and the case that both the IR+XUV pulses are present $\rho_{\mathrm{IRXUV}}$. Considering the time window during which the XUV pulse interacts with the system-which can be resolved within the time resolution of the integration-we subtract the populations obtained with the IR alone and the ones obtained by considering the interaction of both IR + XUV pulses $(\Delta \rho=$ $\left.\rho_{\mathrm{IR}}-\rho_{\mathrm{IRXUV}}\right)$ for each particular $(n, L)$ eigenstate. In order to avoid numerical noise and show cleaner results we set a minimum value of $\Delta \rho$. In the cases that $\Delta \rho<0$ the state is considered "filled" due to the effect of the XUV pulse, since the population of this state at this given time is higher with the presence of the XUV pulse than it was without it. Otherwise, if $\Delta \rho>0$ the state is considered "depleted" by the XUV. We have checked that the states that show changes in the populations in our analysis below also correspond to the states that have most been populated by the presence of the IR field, in every case. Consistently, the effect of the XUV on the populations of the states is a combination of the electric-fieldinduced ac-Stark modulation due to the addition of the oscillating weak XUV field $[33,34]$ together with XUV-stimulated transitions involving both bound and continuum states.

First, our analysis examines weighted histograms resolved in time by considering the population changes $\Delta \rho$ in all states added together. Then we consider selected time windows within the XUV interaction time and calculate weighted histograms of the population differences $\Delta \rho$ determined by the quantum number $n$, the total angular momentum $L$ (with $L_{\max }=300$ ), and the energies of the field-free states $E$ [24].

\section{A. Delay of 3 fs: weak IR field}

The analysis of the distribution of population changes $\Delta \rho$ due to the presence of the XUV pulse corresponding to $\mathrm{H} 21$ at a delay of $t=3.0 \mathrm{fs}$ [see the green line XUV pulse at $3 \mathrm{fs}$ in Fig. 1 and the long-dashed green line in Fig. 5(b)] is shown in Fig. 6. The IR peak intensity is of $6.0 \times 10^{15} \mathrm{~W} / \mathrm{cm}^{2}$ and the 1.5 -fs $\cos ^{2}$ full duration XUV pulse interacts in this case during the time window $2.25<t<3.75$ fs, which corresponds to the values considered in the horizontal axis of Fig. 6(a). By adding up the population differences $\Delta \rho$ of all states at each discretized time (with $\Delta t=14.8$ as) we observe in Fig. 6(a) that the resulting shape basically corresponds to the envelope of the XUV pulse and that there is a clear dynamic switching between more filled [blue (light gray)] and more depleted [red (dark gray)] states, with the periodicity of the XUV field $(\approx 127$ as $)$, as can be expected $[33,34]$. In the panels of Figs. 6(b)-6(d), we study the total XUV interaction time divided in three time windows, as indicated, so that we examine $\Delta \rho$ in the early, center, and late XUV pulse, and compute the histograms as a function of the quantum number $n$ of the states. We see that the populations mostly change in the region $2<n \lesssim 40$, with more effect on the first $2<n \lesssim 6$ states, decreasing at about $n=10$, and having a large contribution 

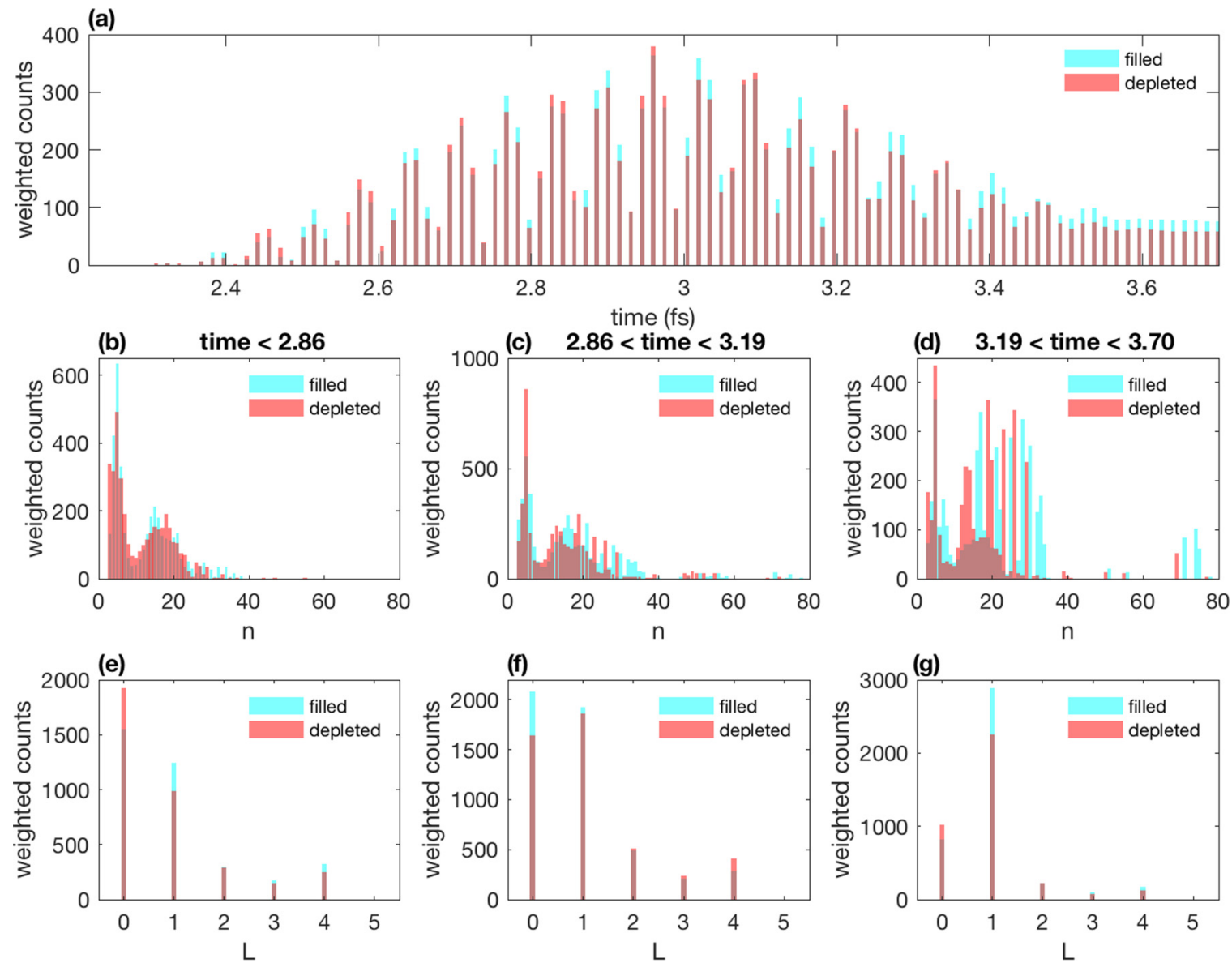

(h)
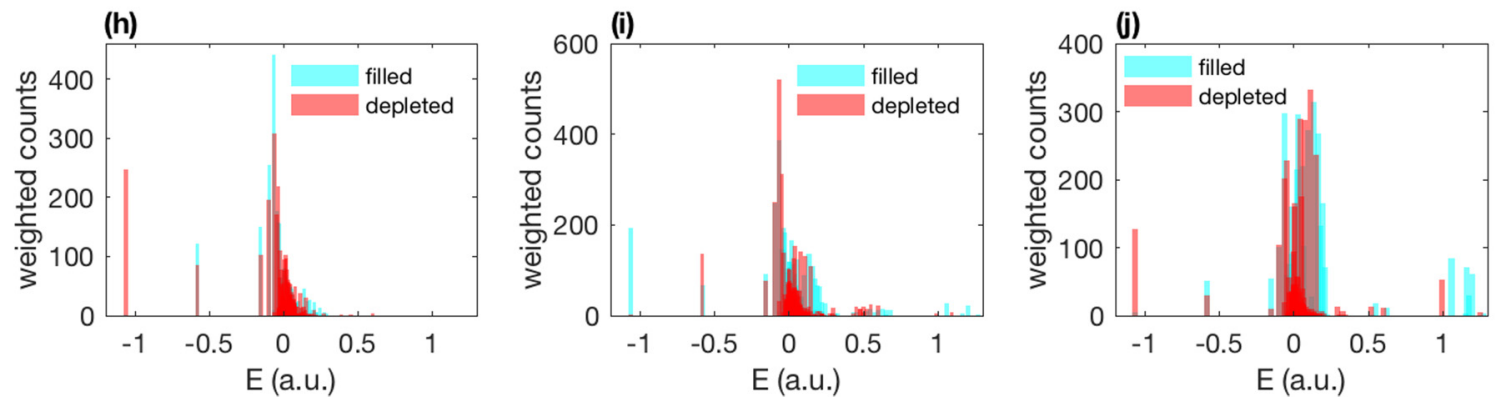

FIG. 6. Weighted histograms of the population differences $\Delta \rho$ in the case of $\mathrm{H} 21$ at a delay of $t=3.0 \mathrm{fs}$ and for an IR peak intensity is of $6.0 \times 10^{15} \mathrm{~W} / \mathrm{cm}^{2}$. As indicated, the histograms are determined by the total number of states in time (a), and by the quantum number $n$ (b)-(d), the angular momentum $L$ (e)-(g), and the energies of the field-free states $E$ (h)-(j) in particular XUV interaction time windows. In (h) $-(\mathrm{j}) E=0$ denotes the transition from bound states to the continuum. The threshold for $\Delta \rho$ is $10^{-8}$ and all vertical axes are divided by this number.

also around $n=20$. In Figs. 6(e) $-6(\mathrm{~g})$ the analysis is shown by resolving the angular momentum $L$. We see that $\Delta \rho$ is concentrated at $L=0$ and $L=1$, and also some higher $1<L<$ 5 angular momentum channels are affected, but in any case always only low $L$ values participate at this delay ( $t=3.0 \mathrm{fs}$ ). The panels in Figs. 6(h)-6(j) show the study performed for the distribution of the population differences $\Delta \rho$ resolved by the eigenenergies of the states, for each selected time window. The involved energies correspond to the IR-excited states both in the bound $(E<0)$ and the continuum $(E>0)$ regions, with $E \lesssim 1$ a.u. We see how the 3 s $(E=-1.07$ a.u. $)$ and 3 p ( $E=$ -0.57 a.u.) states of argon are filled and depleted as the XUV pulse interacts with the atom. These dynamics are correlated with the dynamics in the excited state region about $E=0$, as shown clearly by Figs. 6(h)-6(j), and thus they indicate that the uniform amplification observed for $\mathrm{H} 21$ at the delay of $t=3.0 \mathrm{fs}$ [Fig. 5(b), long-dashed green line] is mainly due to XUV-stimulated transitions from the IR-excited states about $E \geqslant 0$ to the 3 s state with also some less likely contribution of transitions from higher excited states to the $3 p$ state.

\section{B. Delay of 1 fs: strong and moderate IR field}

In Fig. 7 the analysis of $\Delta \rho$ is shown for $\mathrm{H} 21$ at the delay of $t=1.0 \mathrm{fs}$ [see the red line XUV pulse at $1 \mathrm{fs}$ in Fig. 1 and the full red line in Fig. 5(b)]. In this case the temporal 


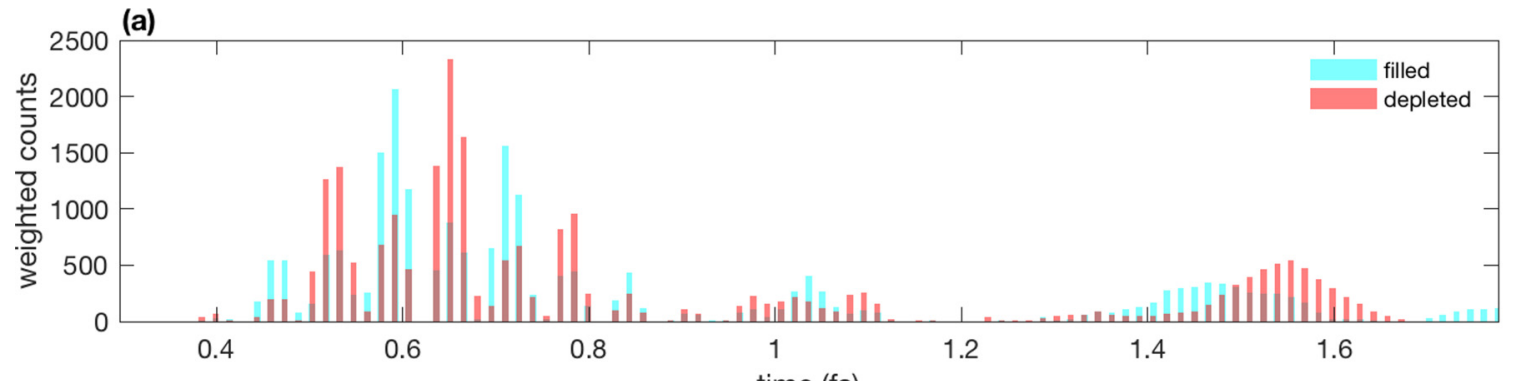

(b) $\quad$ time $<0.90$

(c) $0.90<$ time $<1.20$

(d) $1.20<$ time $<1.78$
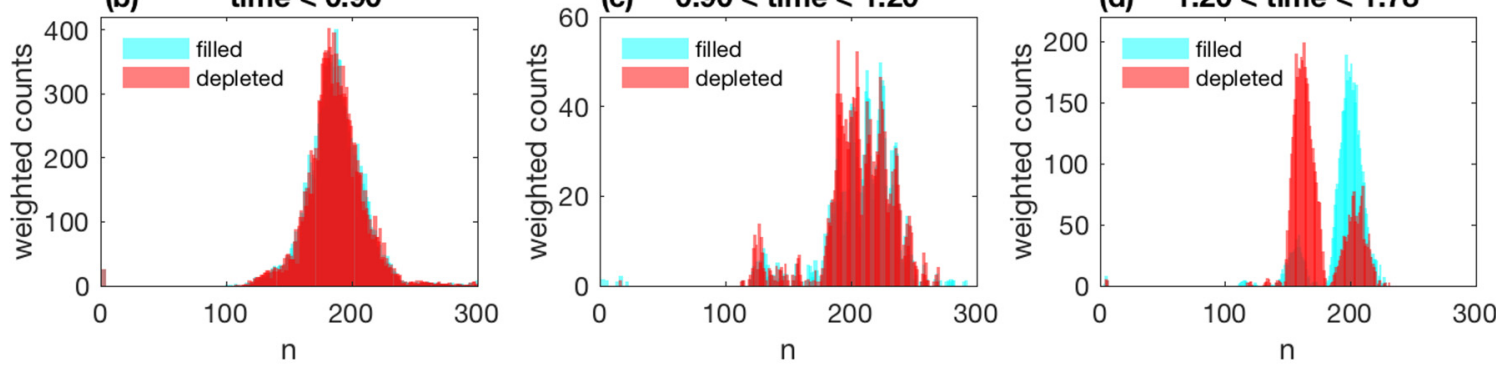

(e)
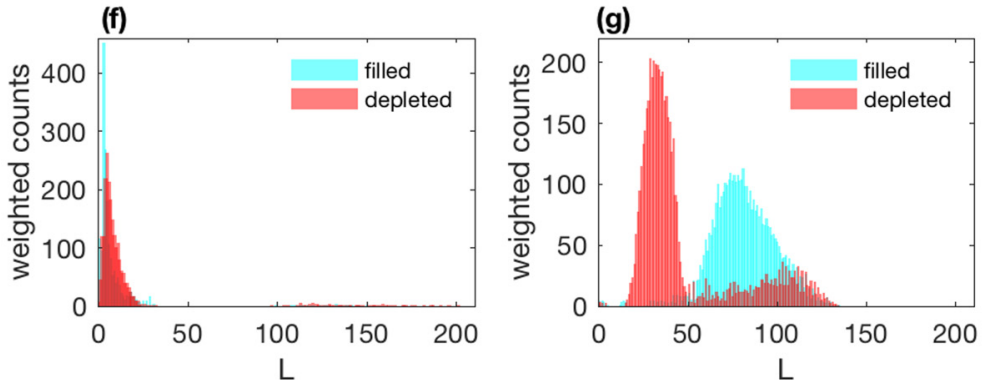

(h)
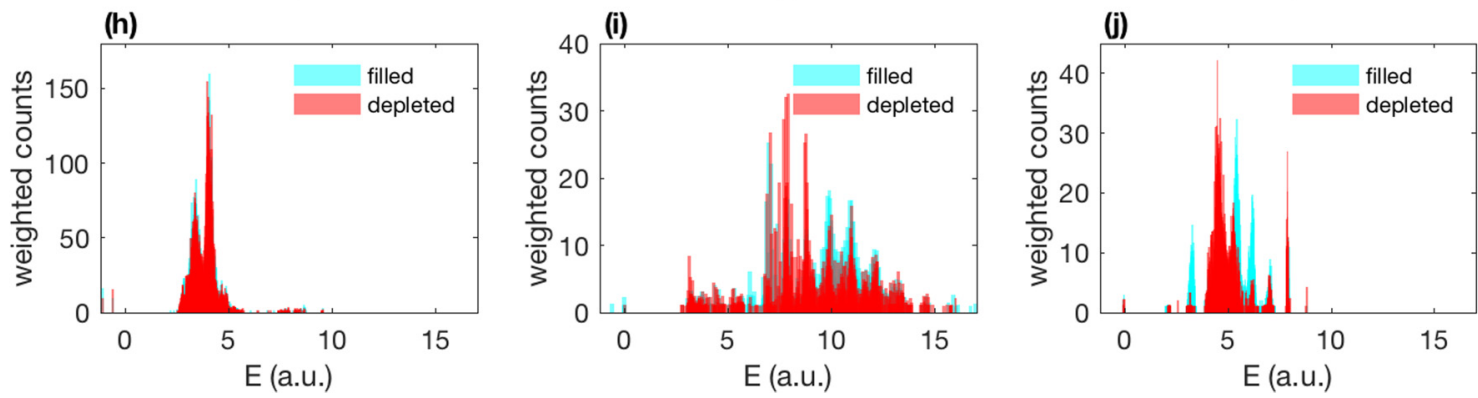

FIG. 7. Weighted histograms of the population differences $\Delta \rho$ in the case of H21 at a delay of $t=1.0$ fs and for an IR peak intensity is of $6.0 \times 10^{15} \mathrm{~W} / \mathrm{cm}^{2}$. As in Fig. 6, the histograms are determined by the total number of states in time (a), and by the quantum number $n$ (b)-(d), the angular momentum $L$ (e)-(g), and the energies of the field-free states $E$ (h)-(j) in particular XUV interaction time windows. In (h) - (j) $E=0$ denotes the transition from bound states to the continuum. The threshold for $\Delta \rho$ is $5 \times 10^{-8}$ and all vertical axes are divided by this number.

window at which the XUV pulse is present $(0.3<t<1.78 \mathrm{fs})$ covers different strengths of the IR electric field, and this fact is reproduced in Fig. 7(a). The IR field has the maximum at $t=0.54 \mathrm{fs}$ and it is zero at $t=1.17 \mathrm{fs}$ (see also Fig. 1). The shape of the histogram hence reproduces $\Delta \rho$ considering the strength of both IR + XUV fields, showing three relative maxima. The switching dynamics between more filled [blue (light gray)] and more depleted [red (dark gray)] states with the periodicity of the XUV field $(\approx 127$ as) is also reproduced in Fig. 7(a), as it was the case in Fig. 6(a). Remarkably, however, we observe that the periodicity changes in the late part of the interaction of the XUV pulse so that the switching involves a somewhat larger period. In the present case we have divided the XUV interaction region by the three time windows at which a substantial effect of the XUV pulse is visible. This is indicated on the top of Figs. 7(b)-7(d). Importantly, in all cases the main changes in the populations occur in states that are in the continuum $(E>0)$. In the left panels, Figs. 7(b), 7(e), and 7(h), the IR field is strong at the early times and moderate at the later, and we see that $\Delta \rho$ includes states $2<n \lesssim 300$ [Fig. 7(b)]. High $n$ quantum numbers are clearly populated in this case with a maximum about $n \approx 190$. The $L$ distribution in Fig. 7(e) also shows most effects in large angular momentum channels $L$, centered about $L=100$. The $E$-resolved panel in Fig. 7(h) shows a maximum distribution of energies about 3 a.u. and 4 a.u. In the bottom 
(a)
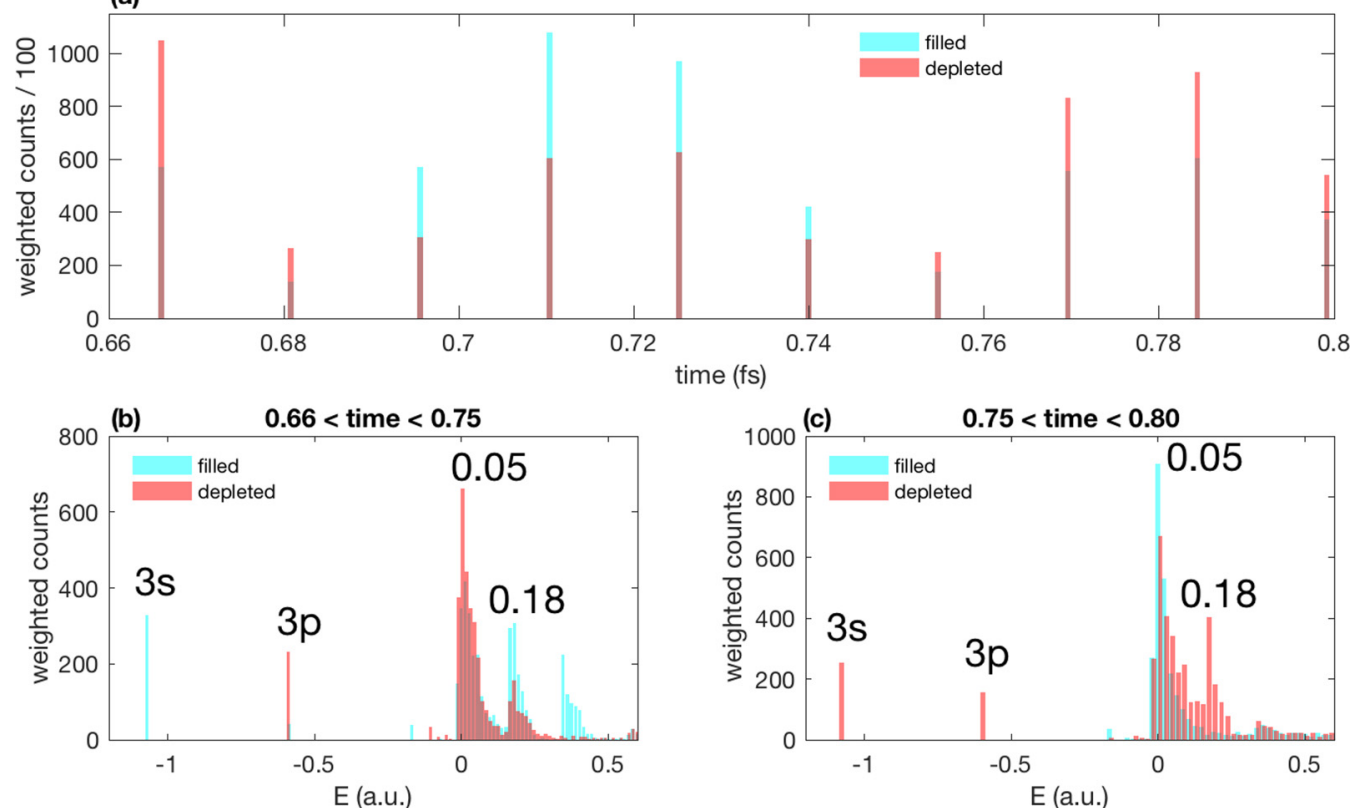

FIG. 8. Weighted histograms of the population differences $\Delta \rho$ in the case of $\mathrm{H} 21$ at a delay of $t=1.0$ fs and for an IR peak intensity is of $6.0 \times 10^{15} \mathrm{~W} / \mathrm{cm}^{2}$ in the XUV interaction time window $0.66<t<0.8 \mathrm{fs}$. The shown histograms are determined by the total number of states in time (a), and by the energies of the field-free states $E$ (b) and (c) in the particular XUV interaction time windows, as indicated. In (b) and (c) $E=0$ denotes the transition from bound states to the continuum. The threshold for $\Delta \rho$ is $2 \times 10^{-9}$ and all vertical axes are divided by this number.

left corner of Fig. 7(h) we can still see a small effect near the ionization potential $(E=0)$ that includes the 3 s and 3 p states, which will be further commented below. The central panels corresponding to the time window $0.9<t<1.2$ fs show a smaller effect because the IR is weaker in this region. The $L$-resolved distribution Fig. 7(f) shows changes in states with a smaller angular momentum $(1<L<30)$. The $E$-resolved histogram [Fig. 7(i)] shows a distribution of energies centered at a larger value than the ones in the left panel [Fig. 7(h)] $\left(E \lesssim 15\right.$ a.u.), which are close to the mean energy $U_{p} \approx$ 13 a.u. for a peak intensity as $6.0 \times 10^{15} \mathrm{~W} / \mathrm{cm}^{2}$ [1]. Also clear in the $\Delta \rho E$-resolved histograms are the peaks separated by $\approx 1.0$ a.u. and also $\approx 0.5$ a.u., which roughly correspond to the ionization energies from the $3 \mathrm{~s}$ and $3 \mathrm{p}$ states. The frequency of the $\mathrm{H} 21(\approx 1.2$ a.u. $)$ is close to this periodicity but its effect is not conclusive from the present simulations and needs to be further examined by considering different XUV central photon energies. In the panels corresponding to the late part of the XUV pulse [Figs. 7(d), 7(g), and $7(\mathrm{j})$ ], we observe that the filling of states is mainly centered at $n=200$ and $L=75$, while states centered at $n=160$ and $L=30$ are mostly depleted. Also, the angular momentum distribution in this case is in between the values in Figs. 7(e) and 7(f), which shows that a higher IR field strength [see also Fig. 1(a)] corresponds to larger $L$ values in the continuum.

We next examine in detail the relatively small changes observed in the low energy region of Fig. 7(h). In order to resolve the dynamics associated with the IR-excited and valence shell states, we set a smaller threshold for $\Delta \rho$, such as $2 \times 10^{-9}$, and zoom this energy region. In particular, Fig. 8 shows the results corresponding to Fig. 7(h) by considering a shorter time window. In the top panel we can observe the $\Delta \rho$ dynamics resolved about the XUV pulse period $(0.66<$ $t<0.8 \mathrm{fs}$ ). In the panels of the bottom [Figs. 8(b) and 8(c)], we can observe correlated population changes between the IR-excited states and the $3 \mathrm{~s}$ and $3 \mathrm{p}$ states. In this case we see a precise structure in the IR-excited states region that shows peaks at 0.05 a.u. and 0.18 a.u., as indicated in the figure. If we now look at the corresponding frequency resolved absorption signal $S(\omega)$, i.e., the full red line in Fig. 5(b), it is evident that it has a peak in absorption at $\approx 1.11$ a.u. $(\approx \mathrm{H} 19.56)$ and a peak in gain at $\approx 1.24$ a.u. $(\approx \mathrm{H} 21.84)$, and it is certainly noticeable that the transitions from the $3 \mathrm{~s}$ state $(-1.064$ a.u.) to these peaked IR-excited states in Figs. 8(b) and 8(c) give 1.11 and 1.24 a.u. The correlated dynamics shown in Figs. 8(b) and 8(c) concerning the fast changes in the populations of the $3 \mathrm{~s}, 3 \mathrm{p}$, and the excited states hence represent a signature of the intra-XUV-parametric processes that were already described in Refs. [15,17], which are now confirmed with 3D-TDSE simulations both in the spectral and in the time domains. The neat IR-excited states structure in Figs. 8(b) and 8(c) was indeed not present in the case of Fig. 6, where the gain was uniform about the spectral domain of the XUV pulse [long-dashed green curve in Fig. 5(b)]. The observed gain hence apparently benefits from the correlated dynamics of bound and continuum states separated in energy by an amount that is comparable with that of the spectral range of the XUV pulse. This occurs in a delay region where the IR field changes significantly from strong to moderate and goes through zero changing sign along the duration of the XUV pulse. This might therefore be the appropriate delay region for the induced parametric processes as reported in Refs. [15,17]. 


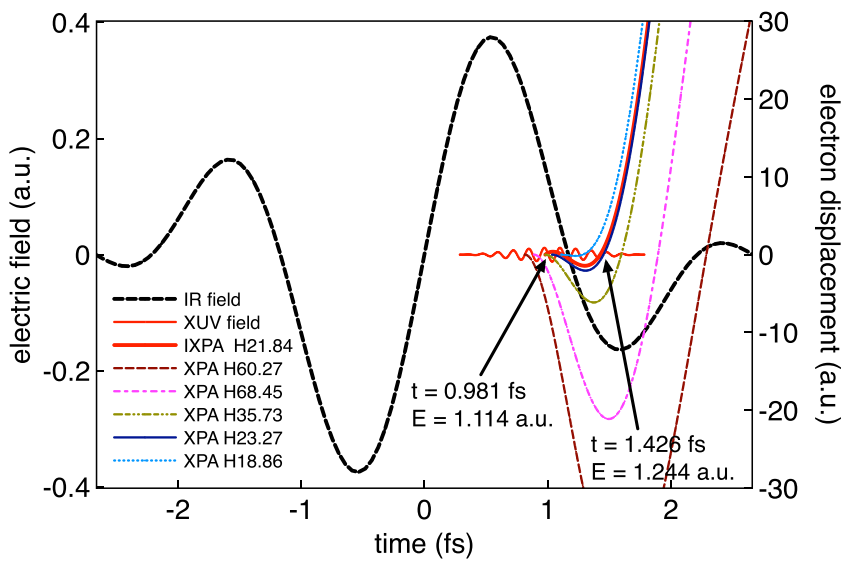

FIG. 9. (Thick full red line) Classical trajectory of an electron ionized from the $3 \mathrm{~s}$ state of argon at $t=0.981$ fs by a photon of energy 1.114 a.u. (H19.56) in a two-cycle IR pulse of $6.0 \times$ $10^{15} \mathrm{~W} / \mathrm{cm}^{2}$. At $t=1.426$ fs the electron can recombine with the $3 \mathrm{~s}$ state with an energy of 1.244 a.u. (H21.84), as indicated, opening the channel for IXPA parametric amplification. (Different styles and colors, thin lines) Electron trajectories of tunnel-ionized electrons that open the channel for XPA parametric amplification. The recombination energies are indicated in terms of (not integer) harmonics.

\section{Semiclassical analysis of the electron trajectories}

To try to further clarify it, we now examine the electron trajectories in the frame of the semiclassical three-step theory [31]. We again take the example of H21 that has been analyzed in Figs. 7 and 8. Specifically, we consider that the interaction of the XUV with the atomic system may be treated in the perturbative regime (i.e., the Keldysh parameter $\gamma=$ $\left.\left.\sqrt{(} 2 I_{p}\right) \omega_{X} / E_{X}>1\right)[21]$. We calculate the classical trajectory of an electron that is ionized from the $3 \mathrm{~s}$ state of $\operatorname{argon}\left(I_{p_{3 s}}=\right.$ 1.064 a.u.) at $t=0.981$ fs by absorbing a single photon in the XUV pulse of energy 1.114 a.u. (H19.56). This value corresponds to the absorption peak in the spectral absorption full red line in Fig. 5(b). We assume that the electron is in the continuum at $x=0$ with velocity $v_{0}^{2}=2\left(1.114-I_{p_{3 s}}\right)$. The electron is then propagated in the two-cycle IR laser field of peak intensity $6.0 \times 10^{15} \mathrm{~W} / \mathrm{cm}^{2}$.

Figure 9 (thick full red line) shows that the classical trajectory crosses twice the atom and that the electron can recombine to the initial $3 \mathrm{~s}$ bound state of the atom in the second crossing at $t=1.426 \mathrm{fs}$; this is with a recombination energy as $E_{\text {rec }}=E_{\text {ret }}+I_{p_{3 s}}=1.244$ a.u. (H21.84, indicated in the legend of the figure), where $E_{\text {ret }}$ is the returning electron's kinetic energy, which corresponds to the emission peak in the spectral absorption full red line in Fig. 5(b). The effect of the Coulomb potential during the trajectory in the continuum and in the rescattering is neglected. Since the returning time is inside the time window of the XUV pulse, the channel for XUVstimulated emission is open. The classical trajectory hence shows how the XUV-stimulated electron ionized at the right time comes back close to the nucleus at the right time and with the right energy, and corroborates that the intra-XUV-pulse (or compulsive) parametric processes reported in Refs. [15,17] are supported by the semiclassical HHG three-step model. Remarkably the XUV-stimulated recombination giving gain about 1.244 a.u. is produced in the late time window of Fig. 7, where the periodicity changes shown in Fig. 7(a) show a switching involving a larger period than the one of the XUV. Otherwise, regular XPA processes are naturally also feasible in this region provided IR-tunnel-ionized electrons come back close to the nucleus at the right times and with the right energies to be recombined through XUV-stimulated emission. Figure 9 (different styles and colors, thin lines) shows some examples of the classical trajectories of electrons that have been ionized by the IR field in the tunneling regime $(\gamma=$ $\left.\left.\sqrt{(} 2 I_{p}\right) \omega_{\mathrm{IR}} / E_{\mathrm{IR}}<1\right)$, i.e., we assume they are at $x=0$ with zero velocity at the ionization time [31]. The recombination energies $E_{\mathrm{rec}}$ in terms of (not integer) harmonics is indicated in the legend of the figure. It is therefore clear that the XPA parametric amplification channel is also open at the different recombination times and photon energies of interest in this delay region.

\section{DISCUSSION AND CONCLUSIONS}

Using a faithful spin-free SAE single-atom theory we perform a rigorous and exhaustive numerical study of the physics in the interaction of strong IR and weak XUV pulses with argon gas in HHG. The $3 \mathrm{~s}$ and $3 p$ ionized regions of argon are consistently analyzed in the frame of the SAE model and the results that we present are in excellent agreement with the gain spectrum measured in the experiments. We report a precise explanation of the observed amplification involving contributions from the electron recombination to the $3 p$ and subvalence 3 s states, with photon energies that are close to the second ionization of argon. Furthermore, we have shown how XUV amplification can indeed be fed by different parametric scattering processes such as IXPA and XPA, and also by the more regular stimulated emission, which decisively depend on the pulse parameters and the medium. Although the values of the calculated gain are somewhat small at the single-atom level, the probability for the parametric amplification effects that we describe is to be exponentially enhanced by propagation in the medium due to avalanche effects as described in Ref. [15].

This work is a step forward in resolving the persistent controversy in the physical interpretation of the scattering mechanisms behind the experimentally observed XUV amplification far from the ionization threshold. In Ref. [16] it was claimed that amplification far from the ionization threshold in helium could not be described by means of the SFA theory used in Refs. [10,14,15]. The SFA numerical simulations, however, identified parametric processes in the basis of the XUV amplification far from the ionization threshold in accordance with the analysis in Refs. [9,12] and had been performed both at the single atom and also considering propagation effects, with great qualitative agreement with the experiments [15]. The SAE 3D-TDSE simulations that we have performed here support the parametric XUV amplification distant from the first ionization threshold in argon as observed in the experiments. In Ref. [19] the need to account for the loss channels in the medium, in particular the one-photon XUV absorption from the ground state to the continuum, together with population inversion, is discussed. Parametric processes are mentioned as candidates 
for the amplification, but they are not studied at all. In the present work we describe in detail which is a clear signature of the parametric contribution in the XUV gain observed in the frame of HHG processes. In Ref. [20] IXPA processes were not achieved using 3D-TDSE simulations in $\mathrm{Li}^{+}$, which were indeed described with a 1D-TDSE model in Ref. [17] and have been now confirmed in argon with 3D-TDSE. Our study comprehends strong, moderate, and weak IR field regions, at which the level of ionization as well as the XUV-induced depletion and population of bounded and continuum states is examined. The analysis that we have performed both in the frequency and in the time domain based on the dynamics of the populations of the eigenstates of the field-free Hamiltonian together with the study of the classical trajectories is an adequate tool to predict other XUV amplification regimes in different pulse and medium geometries. For instance, in the present simulations we have confirmed that intra-XUV-pulse fast dynamics in bound-continuum transitions are significant and induce mixed frequency resolved absorption signals as it was first reported in Refs. [15,17]. Using classical trajectories we have shown that IXPA and XPA channels are open in the region of interest, what explains why the amplification occurs in this case at the final edge of the IR pulse where the field changes sign going from strong to moderate strengths.

Parametric processes such as IXPA and XPA are not blocked by the absence of population inversion in bound- continuum transitions so that the XUV-induced dynamics involving excited states in the continuum that we have outlined makes parametric processes the most plausible candidates for amplification of frequencies far from the ionization threshold, through interaction with largely ionized atoms and the addition of stimulated recombination to innermore states, as we have described in argon. In that perspective, the present results also indicate the convenience of going beyond a spinfree single-electron theory to accurately describe amplification effects as they have been observed, e.g., in argon and helium, in order that contributions from recombinations to all possible ionized states can be incorporated.

\section{ACKNOWLEDGMENTS}

C.S. thankfully acknowledges S. Patchkovskii for the initial instructions on the performance of the code. Computation time was provided by the Super Computer System, Institute for Chemical Research, Kyoto University; Pirineus, Consorci de Serveis Universitaris de Catalunya (CSUC/RES-FI-20183-0003); and JFF-Heat and Mass Transfer Technological Center (CTTC/UPC). Financial support from the Spanish Ministry of Economy and Competitiveness through Grant No. FIS2017-85526-R and the Japan Society for the Promotion of Science (JSPS) (Grant No. JP17H02813) is acknowledged.
[1] P. Agostini and L. F. DiMauro, Rep. Prog. Phys. 67, 813 (2004).

[2] F. Krausz and M. Y. Ivanov, Rev. Mod. Phys. 81, 163 (2009).

[3] A. D. Bandrauk and N. H. Shon, Phys. Rev. A 66, 031401(R) (2002).

[4] K. Ishikawa, Phys. Rev. Lett. 91, 043002 (2003).

[5] K. J. Schafer, M. B. Gaarde, A. Heinrich, J. Biegert, and U. Keller, Phys. Rev. Lett. 92, 023003 (2004).

[6] J. Biegert, A. Heinrich, C. P. Hauri, W. Kornelis, P. Schlup, M. P. Anscombe, M. B. Gaarde, K. J. Schafer, and U. Keller, J. Mod. Opt. 53, 87 (2006).

[7] E. J. Takahashi, T. Kanai, K. L. Ishikawa, Y. Nabekawa, and K. Midorikawa, Phys. Rev. Lett. 99, 053904 (2007).

[8] J. Seres, E. Seres, D. Hochhaus, B. Ecker, D. Zimmer, V. Bagnoud, T. Kuehl, and C. Spielmann, Nat. Phys. 6, 455 (2010).

[9] J. Seres, E. Seres, and C. Spielmann, Phys. Rev. A 86, 013822 (2012).

[10] C. Serrat, Phys. Rev. Lett. 111, 133902 (2013).

[11] F. Brizuela, C. M. Heyl, P. Rudawski, D. Kroon, L. Rading, J. M. Dahlstrom, J. Mauritsson, P. Johnsson, C. L. Arnold, and A. L'Huillier, Sci. Rep. 3, 1410 (2013).

[12] J. Seres, E. Seres, E. Landgraf, B. Ecker, B. Aurand, T. Kuehl, and C. Spielmann, Sci. Rep. 4, 4234 (2014).

[13] J. Seres, E. Seres, E. Landgraf, B. Ecker, B. Aurand, A. Hoffmann, G. Winkler, S. Namba, T. Kuehl, and C. Spielmann, Sci. Rep. 4, 4254 (2014).

[14] C. Serrat, D. Roca, and J. Seres, Opt. Express 23, 4867 (2015).

[15] C. Serrat, D. Roca, J. M. Budesca, J. Seres, E. Seres, B. Aurand, A. Hoffmann, S. Namba, T. Kuehl, and C. Spielmann, Opt. Express 24, 8028 (2016).
[16] T. Bredtmann, S. Patchkovskii, and M. Ivanov, Phys. Rev. Lett. 117, 109401 (2016).

[17] C. Serrat, Phys. Rev. A 93, 063415 (2016).

[18] C. Serrat, Phys. Rev. Lett. 117, 109402 (2016).

[19] T. Bredtmann, S. Patchkovskii, and M. Y. Ivanov, New J. Phys. 19, 073011 (2017).

[20] L. Wang, G.-L. Wang, Z.-H. Jiao, S.-F. Zhao, and X.-X. Zhou, Chin. Phys. B 27, 073205 (2018).

[21] T. S. Sarantseva, M. V. Frolov, N. L. Manakov, A. A. Silaev, N. V. Vvedenskii, and A. F. Starace, Phys. Rev. A 98, 063433 (2018).

[22] H. G. Muller, Phys. Rev. A 60, 1341 (1999).

[23] R. Wiehle, B. Witzel, V. Schyja, H. Helm, and E. Cormier, J. Mod. Opt. 50, 451 (2003).

[24] S. Patchkovskii and H. G. Muller, Comput. Phys. Commun. 199, 153 (2016).

[25] M. V. Ammosov, N. B. Delone, and V. P. Krainov, Zh. Eksp. Teor. Fiz. 91, 2008 (1986) [Sov. Phys. JETP 64, 1191 (1986)].

[26] A. Rudenko, K. Zrost, B. Feuerstein, V. L. B. de Jesus, C. D. Schröter, R. Moshammer, and J. Ullrich, Phys. Rev. Lett. 93, 253001 (2004).

[27] S. Mukamel, in Principles of Nonlinear Optical Spectroscopy (Oxford University Press, Oxford, 1995), p. 103.

[28] Z. Deng and J. H. Eberly, Phys. Rev. Lett. 53, 1810 (1984).

[29] V. Véniard and B. Piraux, Phys. Rev. A 41, 4019 (1990).

[30] C. E. Carroll and F. T. Hioe, Phys. Lett. A 199, 145 (1995).

[31] P. B. Corkum, Phys. Rev. Lett. 71, 1994 (1993).

[32] T. Nubbemeyer, K. Gorling, A. Saenz, U. Eichmann, and W. Sandner, Phys. Rev. Lett. 101, 233001 (2008).

[33] A. Fleischer and N. Moiseyev, Phys. Rev. A 77, 010102(R) (2008).

[34] A. Fleischer, Phys. Rev. A 78, 053413 (2008). 